\title{
O ENVOLVIMENTO DO USUÁRIO NO PROCESSO DE DESENVOLVIMENTO DE ELETROMÉDICOS DE PEQUENAS EMPRESAS
}

Carina Campese (carinacampese @ yahoo.de) - Engenharia de Produção, Universidade de São Paulo

Janaina Mascarenhas Hornos da Costa (janainamhcosta@gmail.com) - Engenharia de Produção, Universidade de São Paulo

\begin{abstract}
RESUMO
A indústria de produtos médicos é regida por uma forte regulamentação que tem o intuito de induzir uma melhoria na qualidade das tecnologias e dos produtos fabricados. $O$ atendimento das normas não assegura, entretanto, a eliminação de todos os erros e perigos associados ao uso desses produtos. Para mitigar alguns desses problemas, é necessário levar em consideração as necessidades e limitações dos diferentes usuários ao longo do desenvolvimento de produtos. A engenharia de usabilidade é encarregada de analisar esse aspecto, sendo a usabilidade definida como a capacidade que um sistema tem de satisfazer as necessidades do usuário. Para garantir uma boa usabilidade de produtos eletromédicos, foi criada em 1977 nos EUA a IEC 60601. No Brasil, a norma referente à usabilidade (NBR IEC 60601-1-6) faz referência ao processo de engenharia de usabilidade da IEC 62366, que conta com seis etapas. Analisando esse contexto surge a seguinte pergunta de pesquisa: "a exigência da norma IEC 60601-1-6 garante que a perspectiva do usuário seja levada em consideração durante o desenvolvimento de produtos eletromédicos?”. Essa pesquisa tem por objetivo compreender como é realizado o envolvimento do usuário e a coleta das suas necessidades no desenvolvimento de produtos de empresas eletromédicos que já estão atendendo à norma de usabilidade. Para tal, estudos de caso exploratórios foram aplicados em quatro empresas de São Carlos e região. Foi identificado que o usuário é envolvido de forma parcial para a coleta das suas necessidades e também na etapa de projeto conceitual. O usuário não é envolvido no desenvolvimento de requisitos e a validação do produto é realizada em diferentes niveis entre as empresas. Esse estudo contribui com um contexto de desenvolvimento de produtos eletromédicos de empresas brasileiras de pequeno porte, servindo como base para futuras pesquisas nessa área.
\end{abstract}

Palavras-chave: Envolvimento do usuário; usabilidade; produtos eletromédicos; desenvolvimento de produtos

Área: O design e o Desenvolvimento de Produtos e Serviços

\section{INTRODUÇÃO}

O mercado de produtos eletromédicos vem crescendo no Brasil (ABIMO, 2014). Segundo essa associação, o país atingiu a segunda colocação de maior produtor dessa área dentre os países em desenvolvimento em 2014. Além disso, o Brasil é o quinto mercado mais atrativo para comercialização de produtos eletromédicos da região das Américas (ALMEIDA et al., 2017).

Essa indústria no Brasil é composta principalmente por pequenas e médias empresas (ALMEIDA et al., 2017), e tem uma forte influência de normas regulatórias (ANVISA, 2014). Essas normas têm o intuito de ajudar e estabelecer requisitos para aprovação dos produtos (ALMEIDA et al., 2017), trazendo uma melhoria na qualidade dos mesmos. 
Entretanto, o atendimento às normas não assegura a eliminação de todos os erros e perigos associados ao uso desses produtos. Rezende, Bernardes e Mello (2015) apontam que 25\% dos erros médicos ocorridos em cirurgias estão relacionados a problemas da tecnologia ou equipamento. Esses erros, ou mesmo mau uso do produto, podem causar sérios problemas ao paciente, ou até mesmo sua morte (ZHANG et al., 2003).

De modo a mitigar esse problema, é preciso que o projeto de produtos seja desenvolvimento levando em consideração as necessidades e limitações dos seus usuários (ULRICH; EPPINGER, 2012), e a engenharia de usabilidade tem a atribuição de dar esse foco ao desenvolvimento de produtos. Entre as normas exigidas para a certificação de produtos eletromédicos, encontra-se a de usabilidade (NBR IEC 60601-1-6), que tem caráter obrigatório desde o final de 2015. No entanto ainda resta a dúvida: a exigência dessa norma garante que a perspectiva do usuário seja levada em consideração durante o desenvolvimento desses produtos? Assim sendo, essa pesquisa tem o objetivo de compreender como é realizado o envolvimento do usuário e como é a coleta das suas necessidades no desenvolvimento de produtos eletromédicos.

\section{USABILIDADE}

Há muitas formas de se definir usabilidade. Para Nielsen (1993), a usabilidade é a capacidade que um sistema tem de satisfazer as necessidades do usuário. Segundo o autor, a usabilidade está tradicionalmente associada a cinco atributos: capacidade de aprendizado (deve ser fácil de se aprender a usar o sistema), eficiência (o uso do sistema deve ser eficiente, se alcançando alta produtividade), memorização (deve ser fácil de se lembrar como usar o sistema), erros (o sistema deve ter uma baixa taxa de erro, senão nenhuma), e satisfação (o sistema deve ser agradável de usar de modo que tornem os usuários satisfeitos).

Já para a ISO 9241-11 (1998), a usabilidade pode ser avaliada sobre quatro aspectos: eficácia (exatidão e integridade com que os usuários atingem seus objetivos, acessando a informação correta ou gerando o resultado esperado), eficiência, satisfação (conforto e aceitabilidade do produto), e contexto de uso (ambiente físico e social no qual o produto é usado), ou seja, a norma se refere à usabilidade também a relacionando ao ambiente no qual o usuário realiza suas atividades, e não somente à forma de como ele as faz. Para lida (2005), a usabilidade está relacionada à facilidade e prevenção e correção de erros no uso de produtos, conforto e eficiência.

\subsection{Benefícios}

Um produto com boa usabilidade pode trazer muitos benefícios tanto para a empresa que o desenvolve quanto para o usuário desse produto. Do ponto de vista empresarial, um produto com boa usabilidade pode trazer os seguintes benefícios:

- Redução no re-design do produto. Quando a usabilidade é aplicada em conjunto a outros processos no desenvolvimento de produtos, não se gasta tempo no fim do desenvolvimento para testes específicos e re-design do produto, quando falhas são encontradas. Com isso, a empresa economiza recursos no desenvolvimento do produto e consegue um lucro inicial do previsto, pois seu produto entrará no mercado antes do que os dos concorrentes (WIKLUND, 2005);

- Redução do risco associado ao produto e redução da incidência de litígios. Com uma boa usabilidade, o produto se torna mais fácil de ser utilizado e também mais seguro. Com isso, ele se torna mais competitivo no mercado, além de a empresa conseguir evitar gastos com indenizações associadas a possíveis danos que o produto pode causar 
(WIKLUND, 2005);

- Redução do custo em treinamento, suporte e assistência. Com a redução do número de reclamações do produto e de dúvidas do consumidor, diminui-se também o número e a duração de ligações e atendimentos, o que reduz custos de operações e infraestrutura (VAN UDEN; RENSINK, 2002; WIKLUND, 2005);

- Melhoria da qualidade do produto final. Quando maior a qualidade do produto, maior a aceitação do mesmo no mercado e maior o número de vendas (VAN UDEN; RENSINK, 2002).

Do ponto de vista do usuário, a usabilidade também traz benefícios:

- Redução do número de erros de utilização. Com uma boa interface, os usuários do produto realizam suas tarefas de forma mais rápida e efetiva, sem ter dúvidas de como utilizar o produto. Isso faz com que a chance de erro de uso no produto diminua, aumentando a produtividade (WIKLUND, 2005) e reduzindo o risco de acidentes (VAN UDEN; RENSINK, 2002);

- Redução / eliminação do estresse físico e mental. Com uma boa percepção de uso do produto, o usuário poupa estresse mental de memória de utilização do mesmo. Além disso, com uma boa usabilidade, é possível operar o produto de forma adequada, evitando possíveis doenças ao usuário (VAN UDEN; RENSINK, 2002).

\subsection{A usabilidade nos produtos eletromédicos}

Em 1977, nos EUA, foi criada a IEC 60601, uma série de normas técnicas para segurança e eficácia de produtos eletromédicos (PEREIRA, 2014). No Brasil, a sua aplicação foi regularizada em 1995 (MORAIS, 2006), e sua norma colateral referente à usabilidade (NBR IEC 60601-1-6) se tornou obrigatória a partir de 2015 (ANVISA, 2014). A NBR IEC 606011-6 especifica um processo para que o fabricante analise, especifique, projete, verifique e valide a usabilidade do produto relacionada à segurança básica e ao desempenho essencial dos produtos eletromédicos.

O processo especificado por essa norma é o processo de engenharia de usabilidade da IEC 62366 (referente a produtos para a saúde), que contém seis etapas, como pode ser observado na figura 1: pesquisa de usuários, projeto conceitual, desenvolvimento de requisitos e critérios, projeto e especificação, avaliação e implantação. Em cada etapa, são requeridas algumas atividades específicas, descritas a seguir. Essas atividades devem ser registradas pela empresa.

Nas etapas de pesquisa de usuários e projeto conceitual (realizadas em conjunto), são exigidas três atividades: (1) especificar aplicação, (2) listar funções frequentemente utilizadas e (3) identificar perigos e situações perigosas relacionadas à usabilidade. A especificação de aplicação deve conter informações de indicação médica do produto, parte do corpo com o qual o produto interage, perfil do usuário, condições de utilização e princípio de operação. Não há orientações específicas na norma sobre o conteúdo da lista de funções utilizadas, mas é preciso seguir um processo de análise de risco estabelecido na ISO 14971 para a identificação das situações perigosas (NBR IEC 62366).

Na etapa de desenvolvimento de requisitos e critérios, são determinadas três atividades: (1) levantar funções primárias de operação, (2) especificar usabilidade e (3) planejar validação de usabilidade. A primeira atividade deve ser feita com base na segunda atividade das etapas anteriores. Já a especificação da usabilidade deve conter requisitos passíveis de ensaio para verificação de usabilidade. O planejamento de validação de usabilidade deve especificar qual 
método será utilizado, quais critérios serão seguidos e a quantos usuários serão envolvidos nessa validação (NBR IEC 62366).

$\mathrm{Na}$ etapa de projeto e especificação detalhados, é exigida somente uma atividade: projetar e implementar a interface do usuário. Para tal, é preciso que o fabricante realize um projeto iterativo. A última etapa antes da aprovação regulamentar (avaliação) demanda duas atividades: (1) verificar usabilidade e (2) validar usabilidade. Pode-se realizar a verificação da usabilidade durante todo o desenvolvimento do produto, já a validação deve ser aplicada na versão final do produto com o envolvimento do usuário (NBR IEC 62366). Após essas etapas, o projeto é submetido para certificação e só a partir dessa aprovação pode ser lançado no mercado.

Dessa forma, fica evidente o envolvimento do usuário no desenvolvimento de produtos eletromédicos nas etapas iniciais (para levantamento do perfil do usuário), na etapa de requisitos (para identificação dos usuários para validação de usabilidade), na etapa final (para a validação da usabilidade) e ao longo do desenvolvimento, para verificação da usabilidade. Além disso, o usuário também pode ser envolvido para levantamento das funções frequentemente utilizadas do produto. Acredita-se que o envolvimento do usuário possa ser efetivo por meio de métodos específicos.

Figura 1 - Processo de engenharia de usabilidade da norma IEC 62366. Fonte: NBR IEC 62366.

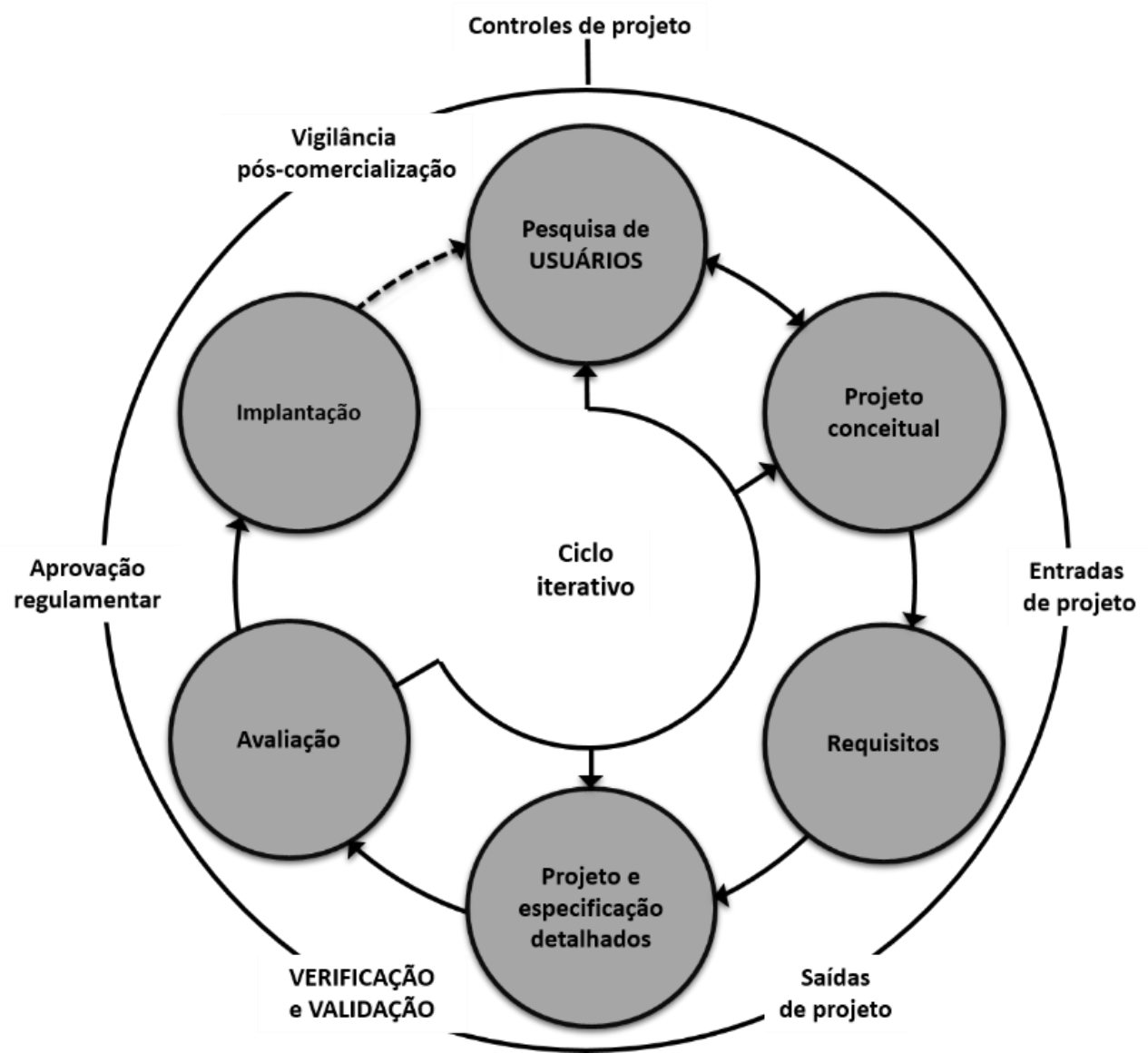

\section{METODOLOGIA}

Foram aplicados nessa pesquisa estudos de caso exploratórios, ou seja, foram analisadas situações contemporâneas dentro de um contexto real (YIN, 2011). Foram realizadas entrevistas em quatro empresas (A, B, C e D) de produtos eletromédicos de pequeno porte na 
região de São Carlos, com um total de seis pessoas entrevistadas, entre elas profissionais da área de projeto e qualidade. Na Tabela 1 encontram-se algumas informações das empresas analisadas.

Tabela 1 - Informações das empresas analisadas. Fonte: elaborada pelas autoras.

\begin{tabular}{llcccc}
\hline Empresa & Linha dos produtos & $\begin{array}{c}\text { Número de } \\
\text { funcionários da } \\
\text { equipe de P\&D }\end{array}$ & $\begin{array}{c}\text { Número de } \\
\text { entrevistados }\end{array}$ & $\begin{array}{c}\text { Duração de } \\
\text { entrevista } \\
\text { (em horas) }\end{array}$ & $\begin{array}{c}\text { Classe de risco } \\
\text { eminente de acidente } \\
\text { com uso do produto }\end{array}$ \\
\hline A & $\begin{array}{l}\text { Oftalmologia, } \\
\text { ginecologia e } \\
\text { otorrinolaringologia }\end{array}$ & 6 & 1 & 1,5 & III (alto risco) \\
B & Oftalmologia & 8 & 3 & 1,5 & $\begin{array}{c}\text { I e II (baixo e médio } \\
\text { risco) }\end{array}$ \\
C & $\begin{array}{l}\text { Neonatologia } \\
\text { Odontologia, } \\
\text { médica geral, }\end{array}$ & 4 & 3 & 1 & III (alto risco) \\
estética & 9 & 1 & 1,5 & III (alto risco) \\
\hline
\end{tabular}

Os estudos de caso exploratórios seguiram um roteiro de perguntas, baseado nas referências estudadas sobre normas e usabilidade. Os dados da identificação das fases e atividades realizadas no desenvolvimento de produtos dessas empresas, assim como a identificação de como é realizada a integração do usuário nesse processo, foram comparados com os dados de fases e envolvimento do usuário que são exigidas pela norma em vigor.

\section{RESULTADOS}

Os resultados das pesquisas nas empresas são apresentados nessa seção separados pelas etapas do processo de engenharia de usabilidade da norma IEC 62366 onde é indicado (de forma direta ou indireta) que haja o envolvimento do usuário. As empresas analisadas possuem desenvolvimento de novos produtos e também projetos de melhorias de produtos que já foram lançados (aprimoramentos). Dessa forma, em cada etapa apresentada nessa seção, são apresentadas as formas de envolvimento do usuário tanto para novos projetos quanto para melhorias.

Foi possível identificar em quais fases do processo de engenharia de usabilidade o usuário é envolvido nas empresas analisadas e também o grau de como esse envolvimento acontece. Devido à obrigatoriedade, todas as empresas analisadas já seguem a norma de usabilidade IEC 60601-1-6.

\section{Pesquisa do usuário e projeto conceitual}

Para a etapa de pesquisa de usuários, a empresa deveria ter um envolvimento com o usuário, já que ela deve apresentar em forma de relatório o perfil do usuário, as condições de utilização do produto e as funções do produto que são frequentemente utilizadas (NBR IEC 62366). Entretanto, foi identificado que somente metade das empresas age dessa maneira.

As empresas A e B realizam o envolvimento com o usuário nessa etapa por meio de entrevistas informais com alguns médicos parceiros da empresa e também com usuários em feiras e congressos nacionais e internacionais. As entrevistas são realizadas para coletar as necessidades desses médicos. Além disso, é realizada também uma pesquisa de mercado. A empresa $\mathrm{C}$ tem um envolvimento com o usuário mais superficial, uma vez que as informações sobre necessidade do mercado são coletadas por pesquisas em editais governamentais e não governamentais de um produto, e pesquisas de produtos concorrentes. Somente após 
estabelecido o novo projeto, são realizadas entrevistas informais com os profissionais da área para entender as suas necessidades. Já a empresa D não realiza esse envolvimento inicial com o usuário, a equipe de desenvolvimento de produtos escolhe o projeto por meio da análise de suas pesquisas de mercado (realizada pela área comercial da empresa).

Todas as empresas analisadas realizam melhorias nos seus produtos após um certo período em que o mesmo está no mercado. O projeto desses produtos passa então por um processo de melhoria que segue novamente as etapas da norma. As empresas A, B e C lidam com o envolvimento do usuário para melhoria do produto da mesma forma como para um novo produto, ou seja, realizam entrevistas informais de modo a identificar como o produto pode ser melhorado. Também acontece de a equipe de projeto implantar modificações sugeridas pela própria equipe, porém foram guardadas porque o processo de certificação do produto já estava em andamento. Já a empresa D realiza suas modificações em projetos baseadas em reclamações pelo SAC e pesquisa de satisfação do cliente por telemarketing (ambas realizadas pela área comercial).

No projeto conceitual, o envolvimento com o usuário também seria possível, uma vez que o usuário pode fazer parte da geração de ideias, sendo envolvido no projeto em um nível maior de interação (MACAULAY, 2012). Nas empresas analisadas, somente a empresa A tem uma sistemática de convidar o usuário para participar da geração de conceitos. Dessa forma, ele tem a possibilidade de sugerir suas ideias de solução para o problema (nem sempre elas são viáveis e são consideradas, mas eles têm essa liberdade e oportunidade). As demais empresas não possuem contato com o usuário para geração de ideias de produto, somente usam dos dados e informações levantadas anteriormente. No caso de melhorias para os produtos, as empresas analisadas se comportam da mesma forma como visto anteriormente, ou seja, somente a empresa A recebe sugestões de conceitos dos usuários.

\section{Requisitos}

Embora não seja direto o envolvimento do usuário, é necessário que se tenha o foco nele nessa etapa para planejar a validação de usabilidade e especificar qual método será aplicado (NBR IEC 62366). Entretanto, foi observado que nessa etapa de desenvolvimento de requisitos somente metade das empresas analisadas possui tal foco, se organizando com os usuários parceiros da empresa para futuros testes com protótipos.

Os requisitos são gerados tendo como base os dados da etapa anterior, tanto para novos projetos quanto para melhorias. Entretanto, somente em metade das empresas analisadas isso ocorre com o apoio de métodos de gestão de requisitos (análise funcional e QFD), ou seja, em metade delas os requisitos são gerados de forma arbitrária.

\section{Avaliação}

Nessa etapa (a última antes de o projeto passar pela aprovação regulamentar), é necessário verificar e validar a usabilidade do produto (NBR IEC 62366). Ressalta-se que a verificação pode ocorrer durante todo o processo de desenvolvimento e que para a validação da versão final do produto, é necessário o envolvimento com o usuário. Na prática, isso não acontece nas empresas analisadas.

A verificação da usabilidade não é realizada em nenhuma das empresas. Os protótipos produzidos em todas as empresas analisadas são $100 \%$ funcionais e fabricados com o mesmo material previsto para o produto final, ou seja, a usabilidade só é verificada quando o produto está praticamente pronto. Já a validação é realizada nas empresas em diferentes níveis. São realizados testes com protótipos em todas as empresas analisadas, porém esses testes não são aplicados da mesma maneira em todas elas. No caso da empresa A, os testes são realizados por médicos parceiros em feiras ou por médicos e universitários em universidades parceiras 
da região, por meio de entrevistas informais. Também é aplicado um teste de usabilidade, realizado na empresa por toda a equipe de $\mathrm{P} \& \mathrm{D}$ e por pelo menos um usuário (médico parceiro). A empresa $\mathrm{B}$ também aplica entrevistas informais com médicos parceiros na própria empresa e/ou em feiras com o uso de protótipos. Na empresa C, os testes com protótipos são realizados pela equipe de projeto, sem nenhum usuário. A empresa $\mathrm{D}$ também não envolve o usuário nesses testes, uma vez que quem os aplica são consultores. Esses resultados de como é o envolvimento do usuário nas etapas do processo de engenharia de usabilidade podem ser observados na Figura 2.

Figura 2 - Envolvimento do usuário nas empresas analisadas. Fonte: elaborada pelas autoras.

\begin{tabular}{|c|c|c|c|c|c|c|}
\hline \multirow{3}{*}{$\begin{array}{l}\text { Etapas do processo de engenharia } \\
\text { de usabilidade da IEC } 62366\end{array}$} & \multicolumn{6}{|c|}{ Envolvimento do usuário } \\
\hline & \multicolumn{2}{|c|}{ Passivo } & \multicolumn{4}{|c|}{ Ativo } \\
\hline & Observação & $\begin{array}{c}\text { Dados } \\
\text { secundários }\end{array}$ & Entrevista & $\begin{array}{l}\text { Teste com } \\
\text { protótipo }\end{array}$ & Brainstorming & $\begin{array}{c}\text { Teste de } \\
\text { usabilidade }\end{array}$ \\
\hline Pesquisa de usuários & O & 0 & ( & $\mathrm{O}$ & 0 & O \\
\hline Projeto conceitual & O & 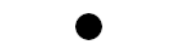 & 0 & 0 & () & O \\
\hline Requisitos & O & 0 & 0 & O & 0 & 0 \\
\hline Projeto e especificação detalhados & $\bigcirc$ & 0 & 0 & O & 0 & 0 \\
\hline Avaliação & 0 & 0 & ( & ( & 0 & $\rightarrow$ \\
\hline
\end{tabular}

Além disso, foi observado que três das empresas analisadas elaboram os relatórios das atividades realizadas somente pouco antes de enviar a documentação para a certificação, e não após o término de cada atividade, como solicitado pela norma.

\section{CONCLUSÕES}

Foi possível identificar que as etapas de pesquisa de usuários e projeto conceitual são realizadas de forma superficial. A maioria das empresas realiza entrevistas com usuários para coleta de dados, porém a empresa tem contato somente com um tipo de usuário (médicos), não levando em consideração os outros tipos de usuários. Isso pode afetar de forma considerável o projeto, uma vez que não se dá atenção a muitas necessidades e requisitos de outros usuários no desenvolvimento dos produtos.

Também foi identificado que a validação dos projetos nem sempre é realizada pelo usuário em si, mas sim pela própria equipe de projeto ou mesmo consultores. Esse fato também pode enviesar o resultado das análises efetuadas.

Além disso, são poucos os métodos que são utilizados que fazem envolvimento do usuário. São aplicados somente os métodos de entrevistas, testes com protótipos, brainstorming e teste de usabilidade, não sendo aplicados necessariamente em todas as empresas. Não é aplicado nenhum método específico para levantar o perfil do usuário (conforme requisitado pela norma) e todas as entrevistas são realizadas de forma informal. Além disso, nenhum método de observação de uso de produto é aplicado para levantamento das funções mais utilizadas para a documentação que a norma exige. Apesar de duas empresas fazerem testes com protótipos, esses não são testados por usuários. Ademais, para o desenvolvimento de requisitos, não foram encontradas evidências de que a equipe de projeto prioriza os requisitos com base no usuário.

Dessa forma, conclui-se que a exigência de aplicação da norma IEC 60601-1-6 não garante que as necessidades do usuário sejam levantadas com o usuário e mesmo levadas em consideração ao longo do desenvolvimento dos produtos eletromédicos, o que pode 
influenciar no resultado final dos equipamentos desenvolvidos e até mesmo não solucionar os problemas de mau uso deles.

\section{REFERÊECIAS}

ABIMO. Brazilian Health Devices. ABIMO em revista. Prêmio Inova Saúde, v. 2, n. 4, p. 22-32, 2014.

ABNT NBR IEC 60601-1-6. Equipamento eletromédico Parte 1-6: requisitos gerais para segurança básica e desempenho essencial - norma colateral: usabilidade. Rio de Janeiro, Brazil, 2013.

ABNT NBR IEC 62366. Produtos para a saúde - Aplicação da engenharia de usabilidade a produtos para a saúde. Rio de Janeiro, 2016.

ALMEIDA, A. P. et al. Framework Proposal Including HFE in Product Development Process : A Suitable Approach for the Brazilian Medical Equipment Industry. Advances in Human Factors and Ergonomics in Healthcare. Springer International Publishing, 2017

ANVISA. Instrução normativa no 4, de 24 de setembro de 2015.

IIDA, I. Ergonomia: projeto e produção. 2a ed. São Paulo: Blucher, 2005.

ISO 9241-11. Ergonomic requirements for office work with visual terminals (VDTs) Part 11: guidance on usability. Switzerland, 1998.

MACAULAY, L. A. Requirements Engineering. Springer Science \& Business Media, 2012.

MORAIS, V. C. Certificação de Produtos para Saúde. ANVISA, 2006.

NIELSEN, J. Usability Engineering. Academic Press, 1993. v. 44

PEREIRA, A. A. Workshop sobre Certificação de Equipamentos Eletromédicos Marco Legal. ANVISA, 2014.

REZENDE, L. S. A.; BERNARDES, M.; MELLO, C. H. P. Engenharia da usabilidade aplicada ao desenvolvimento de equipamentos médicos: uma revisão sistemática. $10 \mathrm{o}$ Congresso Brasileiro de Gestão da Inovação e Desenvolvimento de Produtos. Itajubá - MG: 2015

ULRICH, K. T.; EPPINGER, S. D. Product Design and Development. 5th. ed. McGrawHill Irwin, 2012.

VAN UDEN, M. E. J.; RENSINK, H. J. T. The Development of a Human Factors Engineering Strategy in Petrochemical Engineering and Projects - Part 2. Proceedings at the Occupational Health Offshore Conference. Aberdeen: HSE Books, 2002. Disponível em: <http://www.hse.gov.uk/research/otopdf/2001/oto01041.pdf>

WIKLUND, M. E. Medical Device and Diagnostic Industry. Disponível em: <http://www.mddionline.com/article/return-investment-human-factors $>$. Acesso em: 1 out. 2016.

YIN, R. K. Qualitative Research From Start To Finish. New York: The Guilford Press, 2011.

ZHANG, J. et al. Using usability heuristics to evaluate patient safety of medical devices. Journal of Biomedical Informatics, v. 36, n. 1-2, p. 23-30, 2003. 